\title{
Analysis of Clostridium difficile infections after cardiac surgery: Epidemiologic and economic implications from national data
}

\author{
Andrew Flagg, BS, ${ }^{\mathrm{a}}$ Colleen G. Koch, MD, MS, MBA, ${ }^{\mathrm{b}}$ Nicholas Schiltz, PhD, \\ Aiswarya Chandran Pillai, MD, MS, ${ }^{\mathrm{c}}$ Steven M. Gordon, MD, ${ }^{\mathrm{d}}$ Gösta B. Pettersson, MD, PhD, ${ }^{\mathrm{e}}$ and \\ Edward G. Soltesz, MD, MPH
}

\begin{abstract}
Objectives: Clostridium difficile infections (CDIs) have increased during the past 2 decades, especially among cardiac surgical patients, who share many of the comorbidity risk factors for CDI. Our objectives were to use a large national database to identify the regional-, hospital-, patient-, and procedure-level risk factors for CDI; and determine mortality, resource usage, and cost of CDIs in cardiac surgery.
\end{abstract}

\begin{abstract}
Methods: Using the Nationwide Inpatient Sample database, we identified 349,122 patients who had undergone coronary artery bypass, valve, or thoracic-aortic surgery from 2004 to 2008 . Of these, $2581(0.75 \%)$ had been diagnosed with CDI. Multivariable regression analysis and the propensity method were used for risk adjustment.

Results: Compared with the West, CDIs were more likely to occur in the Northeast (odds ratio [OR], 1.28; 95\% confidence interval [CI], 1.12-1.47) and Midwest (OR, 1.27, 95\% CI, 1.11-1.46) and less likely in the South (OR, 0.80; 95\% CI, 0.70-0.90). Medium-size hospitals (OR, 0.88; 95\% CI, 0.78-0.99) had a lower risk of CDI than did large hospitals. Older age ( $>75$ years; OR, 2.59; $95 \%$ CI, 1.93-3.49), longer preoperative length of stay (OR, 1.51; 95\% CI, 1.43-1.60), Medicare (OR, 1.21; 95\% CI, 1.05-1.39) and Medicaid (OR, 1.60; 95\% CI, 1.31-1.96) coverage, and more comorbidities were associated with CDI. Among the matched pairs, patients with CDIs had greater mortality (302 [12\%] vs $187[7.2 \%], P<.001)$, a longer median length of stay (21 vs 11 days, $P<.001)$, and greater median hospital charges $(\$ 193,330$ vs $\$ 112,245, P<.001)$. The cumulative incremental cost of CDIs was an estimated $\$ 212$ million annually.
\end{abstract}

Conclusions: Our results have shown that CDI is associated with increased morbidity and resource usage. Additional work is needed to better understand the complex interplay among regional-, hospital-, and patient-level factors. (J Thorac Cardiovasc Surg 2014;148:2404-9)

Clostridium difficile infections (CDIs) are increasing at an alarming rate within many hospitalized patient populations, with a parallel increase in the severity of complications, likely because of more virulent strains of the disease. ${ }^{1-4}$ Patients undergoing cardiac surgical procedures share many of the same demographic and comorbidity risk factors associated with an increased risk of CDI. Although population-based studies have clearly documented the poor outcomes of hospitalized patients with $\mathrm{CDI}$ in the absence of any surgical procedures, large-scale

\footnotetext{
From the Department of Biology, ${ }^{\text {a }}$ Case Western Reserve University, Cleveland, Ohio; Department of Cardiothoracic Anesthesia, ${ }^{\mathrm{b}}$ Cleveland Clinic, Cleveland, Ohio; Department of Epidemiology and Biostatistics, ${ }^{\mathrm{c}}$ Case Western Reserve University School of Medicine, Cleveland, Ohio; Department of Infectious Disease, ${ }^{\mathrm{d}}$ Medicine Institute, Cleveland Clinic, Cleveland, Ohio; and Department of Thoracic and Cardiovascular Surgery, ${ }^{\mathrm{e}}$ Heart and Vascular Institute, Cleveland Clinic, Cleveland, Ohio.

Disclosures: Authors have nothing to disclose with regard to commercial support. Received for publication Nov 6, 2013; revisions received March 30, 2014; accepted for publication April 8, 2014; available ahead of print May 10, 2014.

Address for reprints: Edward G. Soltesz, MD, MPH, Department of Thoracic and Cardiovascular Surgery, Heart and Vascular Institute, Cleveland Clinic, 9500 Euclid Ave, Desk J4-1, Cleveland, OH 44195 (E-mail: soltese@ccf.org). $0022-5223 / \$ 36.00$

Copyright (c) 2014 by The American Association for Thoracic Surgery http://dx.doi.org/10.1016/j.jtcvs.2014.04.017
}

epidemiologic data on the morbidity and mortality from secondary development of CDIs after cardiac surgery are lacking.

Previous studies have documented the significantly worse outcomes of patients with CDI in the inpatient surgical setting ${ }^{5}$ and in liver ${ }^{6}$ and solid-organ ${ }^{7}$ transplantation populations. A retrospective study of cardiac surgical patients reported that a longer duration of intensive care unit stay was associated with CDI but not greater mortality. ${ }^{8}$ Because that report was derived solely from the results of 2 large tertiary referral centers in the South, it might not be applicable to the nationwide cardiac surgical population and might not provide an accurate description of patient outcomes in other care settings.

Our objectives were to use a US-derived, nationwide, population-based database to identify the regional-, hospital-, patient-, and procedure-level risk factors for CDIs and determine the excess attributable mortality, resource usage, and cost of CDIs in cardiac surgical patients.

\section{METHODS \\ Data}

An institutional review board-exempt, 5-year period assessment of the Nationwide Inpatient Sample (NIS) Healthcare Cost and Utilization Project database was conducted for 2004 through 2008. The NIS is the largest 


\section{Abbreviations and Acronyms \\ CDI $=$ Clostridium difficile infection \\ CI $=$ confidence interval \\ ICD-9-CM = "International Classification of Diseases, Ninth Revision, Clinical Modification" \\ NIS $=$ Nationwide Inpatient Sample \\ OR $\quad=$ odds ratio}

all-payer inpatient discharge database. The patients include a 20\% stratified sample of all discharges occurring in a given year from approximately 1000 hospitals in 32 to 37 states (depending on the year of the study). The database contains information on about 8 million discharges annually. Individual entries in the database contain information on demographics, comorbidity, hospital charges, length of stay, procedures, outcomes, and discharge. Discharge weights are provided, which allow extraction of nation-level estimates from the unweighted database information.

\section{Population}

The population consisted of 349,122 patients who had undergone cardiac surgical procedures from 2004 to 2008 (this sample extrapolates to $1,709,770$ cardiac surgical procedures in the entire US population). The most common procedures performed were coronary artery bypass grafting ( $\mathrm{n}=246,174$ [71\%]; codes 36.10-36.17, 36.19), isolated valve repair or replacement ( $\mathrm{n}=53,933$ [15\%]; codes 35.10-35.14, 35.20-35.28), isolated thoracic aneurysm repair $(\mathrm{n}=4145[1.2 \%]$; code 38.45$)$, or any combination of these procedures $(n=44,870[13 \%])$. Catheterizations and pacemaker implantations were excluded from the analysis. We examined the demographics and clinical characteristics, primary payer, preoperative comorbidities, surgical procedures, and treating hospital characteristics, such as hospital bed size, teaching status, and regional location, and correlated these with CDI status. The database entries containing "International Classification of Diseases, Ninth Revision, Clinical Modification" (ICD-9$\mathrm{CM}$ ) codes matching these procedures were extracted from the database for patients aged 18 to 100 years. The main exposure of interest was the presence of CDI during admission and was identified by the secondary diagnostic codes (ICD-9-CM code 008.45). The use of ICD-9-CM codes to identify patients with CDI has been validated in other studies, with good accuracy demonstrated. ${ }^{9,10}$

\section{Outcomes}

Our outcomes of interest included hospital mortality, total hospital charges, total costs, length of stay, and discharge disposition. The need for a colectomy procedure was also determined in patients who developed CDI. Colectomy was defined as the presence of 1 of the following ICD-9CM procedure codes: 45.72 to $45.76,45.79$, or $45.8 .^{11}$ The categories of disposition were routine discharge, transferred to another inpatient facility, transfer to another type of facility, and home healthcare. Total charges represented the amount hospitals billed for services and did not include professional fees. Total costs were derived from the total charges using a cost-to-charge ratio created by the Healthcare Cost and Utilization Project in accordance with accounting reports from the Centers for Medicare and Medicaid Services. ${ }^{12}$ Hospital costs will tend to better estimate resource use, because hospital charges often reflect payer policies and other factors unrelated to resource use. The cost was calculated by multiplying the total charges by the hospital-specific cost-to-charge ratio. The costs were adjusted for inflation and converted to 2008 dollars using the appropriate Consumer Price Index.

\section{Covariables}

We considered the demographics, comorbidities, insurance status, hospital characteristics, and region in which the procedures had been performed. The patient-level variables included age, gender, race, and insurance status. Race/ethnicity was categorized as white, black, Hispanic, and other/missing. Certain states do not document race on discharge records; thus, race data were missing for approximately $25 \%$ of the study population. Insurance status was categorized as Medicare, Medicaid, private insurance, uninsured, and other according to the primary payer listed on the discharge record. The comorbidities for risk adjustment were derived from the secondary ICD-9-CM diagnosis codes using the Deyo modification of the Charlson comorbidity index. ${ }^{13,14} \mathrm{~A}$ hospital was considered a teaching hospital if it had an American Medical Association-approved residency program, was a member of the Council of Teaching Hospitals, or had a ratio of full-time equivalent interns and residents to beds of $\geq 0.25$. The hospital region was classified by the US Census Bureau as Northeast, Midwest, South, or West. The hospital bed size was classified as small, medium, or large, using an algorithm developed by the Healthcare Cost and Utilization Project. The surgical procedure was categorized as coronary artery bypass grafting, isolated valve repair or replacement, isolated aneurysm repair, or a combination of $\geq 2$ of these.

\section{Statistical Analysis}

The mean, median, and standard deviation were calculated for all continuous outcomes, and frequency counts and percentages were used for categorical outcomes. Appropriate statistical tests were used for the categorical (chi-square test) and continuous (Student $t$ test) outcomes to compare the unadjusted differences by CDI status. All data included in the present study were unweighted data from the NIS database.

\section{Matching and Outcomes}

Propensity scores, or the conditional probability of having CDI, were estimated for each subject using a multivariable logistic regression model in which the presence of CDI was the dependent variable and patient demographics, payer status, comorbidities, hospital characteristics, and procedure type were the independent variables. ${ }^{15}$ The purpose of the propensity score was to balance the covariables for patients with and without CDI to assess the outcomes without influence from known factors. ${ }^{16}$ To match those with and without CDI, we used a 1:1 greedy matching algorithm with a caliper of 0.2 times the standard deviation of the linear propensity score. ${ }^{17}$ The balance of covariables before and after matching was assessed by calculating the standardized differences between those with and without CDI. ${ }^{18}$ We also performed chi-square tests for all covariables before matching and used McNemar's test in the matched sample. Propensity matching resulted in 2850 well-matched CDI and non-CDI patient pairs with respect to demographics, comorbidities, surgical procedures, and institutional characteristics (ie, region, teaching status; Table 1 and Figure 1).

All analyses were performed using the Statistical Analysis Systems for Unix, version 9.2 (SAS Institute, Inc, Cary, NC).

\section{RESULTS \\ CDI Prevalence}

Of the 349,122 discharge records included in our study, 2581 patients $(0.75 \%)$ had acquired CDI during their hospital stay. The rate of CDI increased from 5.7 to 8.2 cases/1000 patients from 2004 to 2008, peaking during 2007 at 8.4 cases/1000 patients. Patients with and without CDI differed in a number of baseline factors (Figure 1). 
TABLE 1. Distribution of baseline characteristics for propensitymatched patients with and without Clostridium difficile infection

\begin{tabular}{|c|c|c|c|}
\hline Characteristic & $\begin{array}{c}\text { CDI } \\
(\mathbf{n}=\mathbf{2 5 8 0})\end{array}$ & $\begin{array}{c}\text { No CDI } \\
(\mathbf{n}=\mathbf{2 5 8 0})\end{array}$ & $P$ value \\
\hline Age group (y) & & & .7 \\
\hline $0-44$ & $70(2.7)$ & $65(2.5)$ & \\
\hline $45-54$ & $203(7.9)$ & $183(7.1)$ & \\
\hline $55-64$ & $425(16)$ & $405(16)$ & \\
\hline $65-74$ & $814(32)$ & $840(33)$ & \\
\hline$\geq 75$ & $1068(41)$ & $1087(42)$ & \\
\hline Gender & & & .8 \\
\hline Male & $1549(60)$ & $1556(60)$ & \\
\hline Female & $1031(40)$ & $1024(40)$ & \\
\hline Race or ethnicity & & & .8 \\
\hline White & $1594(62)$ & $1624(63)$ & \\
\hline Black & $154(6.0)$ & $141(5.5)$ & \\
\hline Hispanic & $130(5.0)$ & $133(5.2)$ & \\
\hline Other or missing & $702(27)$ & $682(26)$ & \\
\hline Primary payer & & & .5 \\
\hline Medicare & $1788(70)$ & $1829(71)$ & \\
\hline Medicaid & $148(5.7)$ & $121(4.7)$ & \\
\hline Private & $522(20)$ & $510(20)$ & \\
\hline Uninsured & $54(2.1)$ & $51(2.0)$ & \\
\hline Other & $68(2.6)$ & $69(2.7)$ & \\
\hline \multicolumn{4}{|l|}{ Comorbidities } \\
\hline Myocardial infarction & $912(35)$ & $896(35)$ & .6 \\
\hline Heart failure & 1129 (44) & $1158(45)$ & .4 \\
\hline Peripheral arterial disease & $261(11)$ & $259(10)$ & .9 \\
\hline Cerebrovascular disease & $262(10)$ & $230(8.9)$ & .13 \\
\hline COPD & $698(27)$ & $718(28)$ & .5 \\
\hline Rheumatoid disease & $32(1.2)$ & $29(1.1)$ & .7 \\
\hline Peptic ulcer disease & $45(1.7)$ & $38(1.5)$ & .4 \\
\hline Mild liver disease & $11(0)$ & $7(0)$ & .3 \\
\hline Diabetes & $448(17)$ & $442(17)$ & .8 \\
\hline Diabetes and complications & $105(4.1)$ & $103(4.0)$ & .9 \\
\hline Hemiplegia or paraplegia & $36(1.4)$ & $32(1.2)$ & .6 \\
\hline Renal disease & $338(13)$ & $340(13)$ & $>.9$ \\
\hline Cancer & $63(2.4)$ & $62(2.4)$ & $>.9$ \\
\hline Moderate to severe liver disease & $12(0)$ & $6(0)$ & .16 \\
\hline AIDS & $9(0)$ & $6(0)$ & .4 \\
\hline \multicolumn{4}{|l|}{ Surgical procedure } \\
\hline Isolated CABG & $1461(57)$ & $1488(58)$ & .4 \\
\hline Isolated valve repair & $518(20)$ & $492(19)$ & .4 \\
\hline Isolated aneurysm repair & $76(2.9)$ & $69(2.7)$ & .6 \\
\hline Combination surgery & $525(20)$ & $531(21)$ & .8 \\
\hline \multicolumn{4}{|l|}{ Hospital characteristics } \\
\hline Region & & & .8 \\
\hline Northeast & $719(28)$ & $719(28)$ & \\
\hline Midwest & $626(24)$ & $611(24)$ & \\
\hline South & $798(31)$ & $829(32)$ & \\
\hline West & 437 (17) & $421(16)$ & \\
\hline Teaching status & & & $>.9$ \\
\hline Teaching & $1738(67)$ & $1737(67)$ & \\
\hline Nonteaching & $842(33)$ & 843 (33) & \\
\hline Hospital bed size & & & .3 \\
\hline Small & $171(7)$ & $149(5.8)$ & \\
\hline Medium & $412(16)$ & $392(15)$ & \\
\hline Large & 1997 (77) & $2039(79)$ & \\
\hline
\end{tabular}

Data presented as n (\%).CDI, Clostridium difficile infection; COPD, chronic obstructive pulmonary disease; $C A B G$, coronary artery bypass grafting; AIDS, acquired immunodeficiency syndrome.

\section{Predictors}

We found a number of predictors of CDI in the cardiac surgical setting. Regional differences were present, with a greater incidence of CDI in the Northeast (odds ratio [OR], 1.28; 95\% confidence interval [CI], 1.12-1.47) and Midwest (OR, 1.27; 95\% CI, 1.11-1.46) than in the South and a greater risk in teaching hospitals (OR, 1.14; 95\% CI, 1.04-1.26). Patients with Medicare and Medicaid insurance had greater odds of developing CDI (OR, 1.21; 95\% CI, 1.05-1.39; and OR, 1.60; 95\% CI, 1.31-1.96; respectively) than patients with private insurance. Of the surgical procedures, patients who had undergone isolated coronary artery bypass grafting were less likely to develop CDI (OR, 0.69; 95\% CI, 0.61-0.78) than those undergoing other procedures. Most cardiac surgical procedures were performed in large (urban hospitals with $\geq 325-450$ beds; two thirds of patients were treated in teaching hospitals; Table 2).

Patient demographics such as older age, female gender, longer preoperative hospital length of stay, and greater comorbidity burden (eg, heart failure, chronic obstructive pulmonary disease, acute myocardial infarction, and liver disease) were associated with an increased prevalence of CDI. Other factors that increased the risk included emergency surgery and hospital transfers (Table 2).

\section{Matching and Outcomes}

Patients with and without CDI had a high comorbidity burden, which is common in those undergoing cardiac surgical procedures. Unadjusted outcomes are presented in Table 3. The propensity-adjusted outcomes for these patients are listed in Table 4. The median length of stay for those who developed CDI was nearly double that of those who did not ( 21 vs 11 days, $P<.001$ ), hospital mortality was significantly greater $(\mathrm{n}=302[12 \%]$ vs $\mathrm{n}=187$ [7.2\%], $P<.001)$, and the total median charges $(\$ 193,330$ vs $\$ 112,245, P<.001)$ and hospital costs $(\$ 65,809$ vs $\$ 39,094, P<.001)$ were greater. Among the patients with CDI, 69 (2.7\%) required subsequent colectomy during the same hospital stay. Also, significant differences were found in the discharge disposition, with the patients with CDI more commonly transferred to other inpatient care facilities $(\mathrm{n}=1246[48 \%]$ vs $775[15 \%], P<.001$, respectively).

For all cardiac surgical patients in the United States, the incremental average cost annually for CDI was estimated at $\geq \$ 212$ million according to the weighted estimate of patients with CDI $(\mathrm{n}=12,772[0.75 \%])$ for the 5 -year period (Table 5).

\section{DISCUSSION}

We report the regional-, local-, and patient-specific variation in the risk of developing CDI after cardiac surgical 


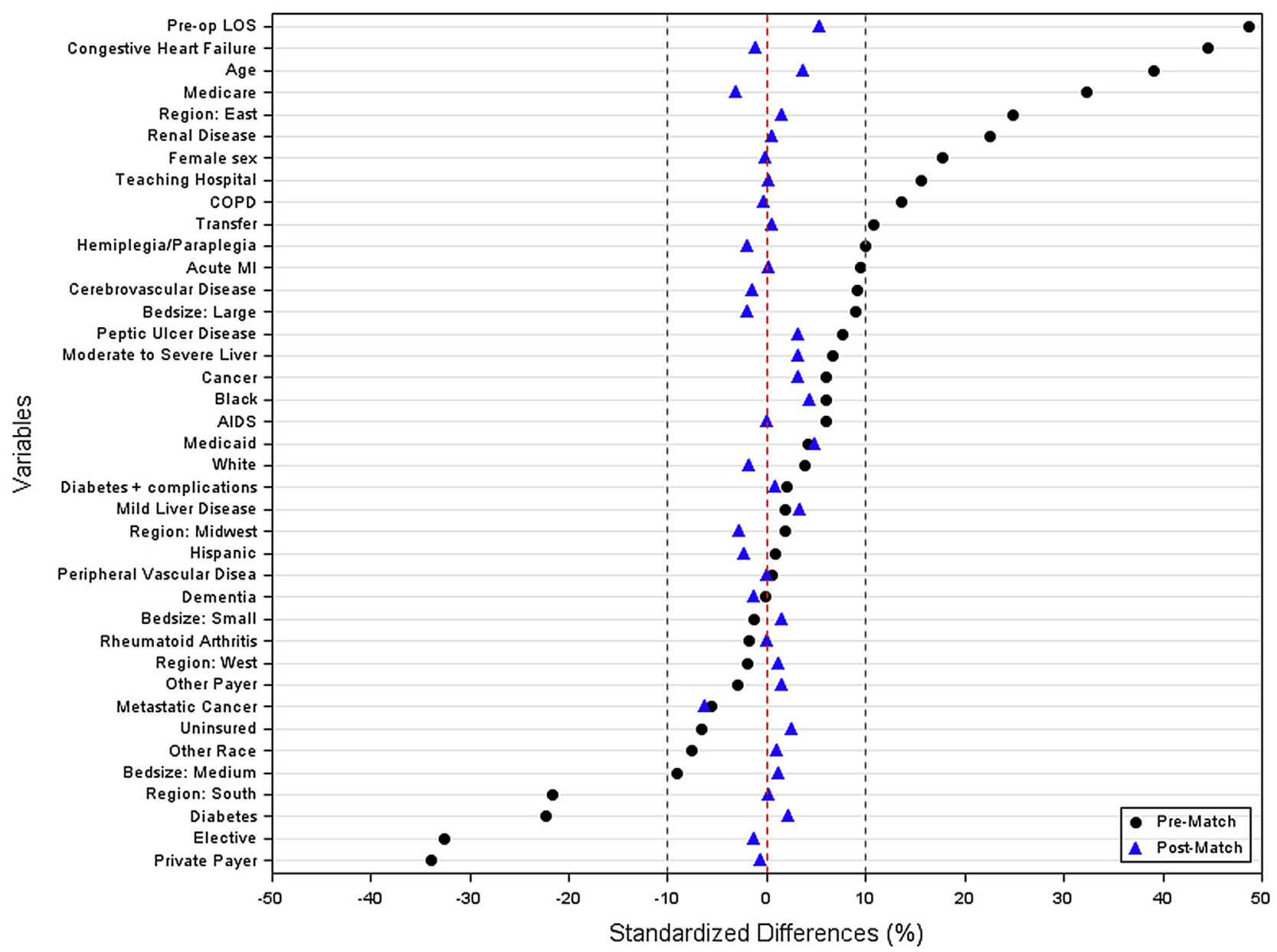

FIGURE 1. Covariance balance plot for patients with and without Clostridium difficile infections before and after propensity matching. LOS, Length of stay; $C O P D$, chronic obstructive pulmonary disease; $M I$, myocardial infarction; $A I D S$, acquired immunodeficiency syndrome.

procedures. For those who acquired CDI, mortality was increased, the associated costs of care were greater, and the need for more intensive services in the follow-up period was greater. Our findings of regional and local variation in the occurrence of CDI, with a heightened risk for procedures performed in the Northeast and Midwest and in large, urban, and/or teaching hospitals, suggest the need for more intense examination of regional practices and an opportunity for process improvement.

A recent large hospital-based study in Barcelona, Spain, found risk factors for CDI consistent with our results. That epidemiologic study, which examined 365 episodes of CDI, found significant risk associated with advanced patient age and a high comorbidity score. ${ }^{19}$ Previous studies have found links between CDI and mortality and morbidity in scenarios other than cardiac surgery. In a study using NIS data to examine patients who had undergone liver transplantation from 2004 to 2008, Ali and colleagues ${ }^{6}$ identified CDI as an independent risk factor for mortality. Again, comorbidities and advanced patient age were risk factors for CDI.
Another NIS database study by Pant and colleagues ${ }^{7}$ in a solid-organ transplant population reported increased mortality, length of stay, and hospital charges for patients who developed CDI.

In contrast to our investigation, a 2007 study by Crabtree and colleagues ${ }^{8}$ found similar risk factors for CDI in cardiac surgical patients but no increase in 30-day mortality. Similarly, a study involving 151 lung transplant recipients did not identify a significant increase in mortality or any significant risk factors for the 7 patients who had developed CDI during their initial hospitalization. ${ }^{20}$

In addition to patient health burden, the economic burden of CDI on cardiac surgical patients is great, with an estimated cumulative incremental cost of $\$ 212$ million annually. An important aspect of our findings was the large incremental cost of CDI to patients undergoing cardiac surgery. Zerey and colleagues ${ }^{5}$ analyzed all inpatient records in the NIS from 1999 to 2003 and made a related observation, finding a mean $\$ 77,483$ increase in total hospital charges for patients with CDI. 
TABLE 2. Predictors of acquiring postoperative Clostridium difficile infection

\begin{tabular}{|c|c|}
\hline Variable & Adjusted OR $(95 \%$ CI \\
\hline \multicolumn{2}{|l|}{ Age group (y) } \\
\hline $0-44$ & Referent \\
\hline $45-54$ & $1.19(0.88-1.62)$ \\
\hline $55-64$ & $1.41(1.06-1.88)$ \\
\hline $65-74$ & $2.03(1.51-2.72)$ \\
\hline$\geq 75$ & $2.59(1.93-3.49)$ \\
\hline Female gender & $1.18(1.08-1.29)$ \\
\hline Nontransfers & $0.85(0.76-0.94)$ \\
\hline Elective & $0.78(0.71-0.87)$ \\
\hline Preoperative length of stay & $1.51(1.43-1.60)$ \\
\hline \multicolumn{2}{|l|}{ Race or ethnicity } \\
\hline White & Referent \\
\hline Black & $1.16(0.96-1.39)$ \\
\hline Hispanic & $1.11(0.92-1.34)$ \\
\hline Other or missing & $0.98(0.88-1.09)$ \\
\hline \multicolumn{2}{|l|}{ Primary payer } \\
\hline Private & Referent \\
\hline Medicare & $1.21(1.05-1.39)$ \\
\hline Medicaid & $1.60(1.31-1.96)$ \\
\hline Uninsured & $1.00(0.73-1.38)$ \\
\hline Other & $1.33(1.01-1.77)$ \\
\hline \multicolumn{2}{|l|}{ Comorbidities } \\
\hline Myocardial infarction & $1.13(1.02-1.24)$ \\
\hline Heart failure & $1.76(1.60-1.92)$ \\
\hline Peripheral arterial disease & $0.86(0.73-1.01)$ \\
\hline Cerebrovascular disease & $1.15(0.99-1.32)$ \\
\hline Dementia & $0.58(0.14-2.34)$ \\
\hline COPD & $1.23(1.12-1.36)$ \\
\hline Rheumatoid disease & $0.68(0.45-1.02)$ \\
\hline Peptic ulcer disease & $1.47(1.06-2.05)$ \\
\hline Mild liver disease & $0.79(0.39-1.60)$ \\
\hline Diabetes & $0.64(0.57-0.71)$ \\
\hline Diabetes and complications & $0.82(0.66-1.02)$ \\
\hline Hemiplegia or paraplegia & $2.11(1.40-3.19)$ \\
\hline Renal disease & $1.49(1.30-1.71)$ \\
\hline Cancer & $1.29(0.98-1.70)$ \\
\hline Moderate to severe liver disease & $3.47(1.83-6.59)$ \\
\hline AIDS & $3.93(1.90-8.11)$ \\
\hline \multicolumn{2}{|l|}{ Surgical procedure } \\
\hline Isolated CABG & $0.69(0.61-0.78)$ \\
\hline Isolated valve repair & $1.00(0.87-1.15)$ \\
\hline Isolated aneurysm repair & $2.93(2.17-3.95)$ \\
\hline \multicolumn{2}{|l|}{ Hospital characteristics } \\
\hline \multicolumn{2}{|l|}{ Region } \\
\hline Northeast & $1.28(1.12-1.47)$ \\
\hline Midwest & $1.27(1.11-1.46)$ \\
\hline South & $0.80(0.70-0.90)$ \\
\hline West & Referent \\
\hline Teaching hospital & $1.14(1.04-1.26)$ \\
\hline \multicolumn{2}{|l|}{ Hospital bed size } \\
\hline Small & $1.12(0.95-1.32)$ \\
\hline Medium & $0.88(0.78,0.99)$ \\
\hline Large & Referent \\
\hline
\end{tabular}

Total patients, 349,122; total cases of CDI, 2581. OR, Odds ratio; $C I$, confidence interval; $C O P D$, chronic obstructive pulmonary disease; $A I D S$, acquired immunodeficiency syndrome; $C A B G$, coronary artery bypass grafting.
TABLE 3. Unadjusted outcomes for patients with and without Clostridium difficile infection

\begin{tabular}{lccc}
\hline \multicolumn{1}{c}{ Variable } & $\begin{array}{c}\text { CDI } \\
(\mathbf{n}=\mathbf{2 5 8 1})\end{array}$ & $\begin{array}{c}\text { No CDI } \\
(\mathbf{n}=\mathbf{3 4 6 , 5 4 1})\end{array}$ & $\boldsymbol{P}$ value \\
\hline Median length of stay (d) & 21 & 8 & \\
Median total charges (US\$) & $\$ 193,255$ & $\$ 88,736$ & \\
Median total cost (US\$) & $\$ 65,799$ & $\$ 31,125$ & \\
Hospital mortality & $302(12)$ & $11,220(3.2)$ & $<.0001$ \\
Discharge disposition & & & $<.0001$ \\
$\quad$ Home healthcare & $577(22)$ & $110,660(32)$ & \\
$\quad$ Inpatient facility & $1246(48)$ & $62,794(18)$ & \\
$\quad$ Other transfer & $306(12)$ & $11,532(3.3)$ & \\
$\quad$ Routine/home transfer & $447(17)$ & $161,264(47)$ & \\
\hline Data presented as n (\%), unless otherwise noted. CDI, Clostridium difficile infection.
\end{tabular}

\section{Study Limitations}

We recognize that our study had several limitations. The present study made use of a large administrative database with the potential for under- or overdiagnosis and coding errors. The incidence of CDI might have been underestimated, because data in the NIS database is limited to a patient's hospital stay. CDI could certainly have been diagnosed after a patient's discharge. The results of toxin assays and other tests used to diagnose CDI are unavailable in the NIS, and the coding does not differentiate hospital-acquired CDI from preoperative CDI. Nonetheless, several studies have confirmed that ICD-9-CM codes can be an acceptable surrogate to track CDI, with a pooled estimated positive predictive value of $87 \%$ and negative predictive value of $99.7 \%{ }^{21}$ Although Dubberke and colleagues ${ }^{10}$ have found that the use of the CDI ICD-9-CM code without the "present on admission" flag cannot distinguish between hospital-acquired from community-acquired disease states, we believe that the rate of community-acquired CDI in the cardiac surgery population will be very low because the presence of CDI would likely postpone cardiac surgery in most instances. The duration of antibiotic use is an important risk factor for the development of CDI, but the NIS does not provide data on antibiotic type or duration of use. Although we were are to perform a robust risk

TABLE 4. Risk-adjusted outcomes among propensity-matched pairs

\begin{tabular}{lccr}
\hline \multicolumn{1}{c}{ Variable } & $\begin{array}{c}\text { CDI } \\
(\mathbf{n = 2 5 8 0})\end{array}$ & $\begin{array}{c}\text { No CDI } \\
(\mathbf{n = 2 5 8 0})\end{array}$ & $\boldsymbol{P}$ value \\
\hline Median length of stay (d) & 21 & 11 & $<.001$ \\
Median total charges (US\$) & $\$ 193,330$ & $\$ 112,245$ & $<.001$ \\
Median total cost (US\$) & $\$ 65,809$ & $\$ 39,094$ & $<.001$ \\
Mortality & $302(12)$ & $187(7.2)$ & $<.001$ \\
Disposition & & & $<.001$ \\
$\quad$ Home healthcare & $577(22)$ & $836(32)$ & \\
Inpatient facility & $1246(48)$ & $775(15)$ & \\
Other transfer & $309(12)$ & $189(7.3)$ & \\
$\quad$ Routine or home transfer & $446(17)$ & $778(30)$ & \\
\hline
\end{tabular}

Data presented as n (\%), unless otherwise noted. CDI, Clostridium difficile infection. 
TABLE 5. Total and average cost annually for cardiac surgical patients with Clostridium difficile infection

\begin{tabular}{lrcc}
\hline \multicolumn{1}{c}{ Year } & Total cost (US\$) & $\begin{array}{c}\text { Median } \\
\text { cost } \mathbf{( U S \$ )}\end{array}$ & $\begin{array}{c}\text { 15th-85th } \\
\text { percentile }\end{array}$ \\
\hline 2004 & $\$ 145,069,123$ & $\$ 57,861$ & $\$ 30,587-120,679$ \\
2005 & $\$ 203,069,363$ & $\$ 63,660$ & $\$ 34,244-137,653$ \\
2006 & $\$ 217,922,600$ & $\$ 61,398$ & $\$ 32,244-129,781$ \\
2007 & $\$ 234,752,447$ & $\$ 70,147$ & $\$ 37,626-151,942$ \\
2008 & $\$ 262,275,350$ & $\$ 76,875$ & $\$ 39,915-164,819$ \\
$2004-2008$ & $\$ 1,063,088,883$ & - & - \\
Average annual cost & $\$ 212,617,777$ & - & - \\
\hline
\end{tabular}

adjustment using the Deyo modification of the Charlson comorbidity index, certain key covariates such as the degree of frailty and severity of comorbidities are not available in the NIS. Despite these limitations, our study leveraged the large number of patients in the NIS database to evaluate the association of CDI with the outcomes in cardiac surgery patients, controlling for potential confounding variables, including demographics, comorbidities, institution, and payer.

\section{CONCLUSIONS}

Our findings suggest that location is an important factor in the development of CDI. Variations in the prevalence of CDI both regionally and locally suggest an opportunity for process improvement in high-risk locations. The occurrence of CDI infections is also related to patient characteristics and is associated with greater mortality and increased resource usage in the cardiac surgical setting. Additional work is needed to better understand the transmission of $\mathrm{CDI}$, the role of potentially modifiable risk factors, and the key features specific to regional hospitals that appear to increase the risk.

The authors wish to thank Tess Parry for her valuable help in editing our report.

\section{References}

1. Khanna S, Pardi DS. The growing incidence and severity of Clostridium difficile infection in inpatient and outpatient settings. Expert Rev Gastroenterol Hepatol. 2010;4:409-16

2. Jawa RS, Mercer DW. Clostridium difficile-associated infection: a disease of varying severity. Am J Surg. 2012;204:836-42.
3. Ryan K, Ray C, eds. Sherris Medical Microbiology. 5th ed. New York: McGraw Hill; 2011.

4. Gerding DN, Johnson S, Peterson LR, Mulligan ME, Silva J Jr. Clostridium difficile-associated diarrhea and colitis. Infect Control Hosp Epidemiol. 1995;16: 459-77.

5. Zerey M, Paton BL, Lincourt AE, Gersin KS, Kercher KW, Heniford BT. The burden of Clostridium difficile in surgical patients in the United States. Surg Infect (Larchmt). 2007;8:557-66.

6. Ali M, Ananthakrishnan AN, Ahmad S, Kumar N, Kumar G, Saeian K. Clos tridium difficile infection in hospitalized liver transplant patients: a nationwide analysis. Liver Transpl. 2012;18:972-8.

7. Pant C, Anderson MP, O'Connor JA, Marshall CM, Deshpande A, Sferra TJ. Association of Clostridium difficile infection with outcomes of hospitalized solid organ transplant recipients: results from the 2009 Nationwide Inpatient Sample database. Transpl Infect Dis. 2012;14:540-7.

8. Crabtree T, Aitchison D, Meyers BF, Tymkew H, Smith JR, Guthrie TJ, et al. Clostridium difficile in cardiac surgery: risk factors and impact on postoperative outcome. Ann Thorac Surg. 2007;83:1396-402.

9. Dubberke ER, Butler AM, Nyazee HA, Reske KA, Yokoe DS, Mayer J, et al. The impact of ICD-9-CM code rank order on the estimated prevalence of Clostridium difficile infections. Clin Infect Dis. 2011;53:20-5.

10. Dubberke ER, Butler AM, Yokoe DS, Mayer J, Hota B, Mangino JE, et al. Multicenter study of surveillance for hospital-onset Clostridium difficile infection by the use of ICD-9-CM diagnosis codes. Infect Control Hosp Epidemiol. 2010; $31: 262-8$.

11. Lesperance K, Causey MW, Spencer M, Steele SR. The morbidity of Clostridium difficile infection after elective colonic resection-results from a national population database. Am J Surg. 2011;201:141-8.

12. Healthcare Cost and Utilization Project (HCUP). HCUP Clinical Classification Software and Services and Procedures. Agency for Healthcare Research and Quality, Rockville, MD. Available at: http://wwwhcup-usahrqgov/ toolssoftware/ccs_sccsproc/ccssvcprocjsp. Accessed June 1, 2013.

13. Quan H, Sundararajan V, Halfon P, Fong A, Burnand B, Luthi JC, et al. Coding algorithms for defining comorbidities in ICD-9-CM and ICD-10 administrative data. Med Care. 2005;43:1130-9.

14. Deyo RA, Cherkin DC, Ciol MA. Adapting a clinical comorbidity index for use with ICD-9-CM administrative databases. J Clin Epidemiol. 1992;45:613-9.

15. Rosenbaum PR, Rubin DB. The central role of the propensity score in observational studies for causal effects. Biometrika. 1983;70:41-55.

16. Rosenbaum $P$, Rubin D. Constructing a control group using multivariate matched sampling methods that incorporate the propensity score. Am Stat. 1985;39:33-8.

17. Parsons LS. Reducing bias in a propensity score matched-pair sample using greedy matching techniques. Proceedings of the Twenty-Sixth Annual SAS Users Group International Conference; 2001. Cary, NC: SAS Institute, Inc; 2001:214-26.

18. Austin PC, Grootendorst P, Anderson GM. A comparison of the ability of different propensity score models to balance measured variables between treated and untreated subjects: a Monte Carlo study. Stat Med. 2007;26:734-53.

19. Rodriguez-Pardo D, Almirante B, Bartolomé RM, Pomar V, Mirelis B Navarro F, et al. Epidemiology of Clostridium difficile infection and risk factors for unfavorable clinical outcomes: results of a hospital-based study in Barcelona, Spain. J Clin Microbiol. 2013;51:1465-73.

20. Lee JT, Hertz MI, Dunitz JM, Kelly RF, D'Cunha J, Whitson BA, et al. The rise of Clostridium difficile infection in lung transplant recipients in the modern era. Clin Transplant. 2013;27:303-10.

21. Goto M, Ohl ME, Schweizer ML, Perencevich EN. Accuracy of administrative code data for the surveillance of healthcare-associated infections: a systematic review and meta-analysis. Clin Infect Dis. 2014;58:688-96. 